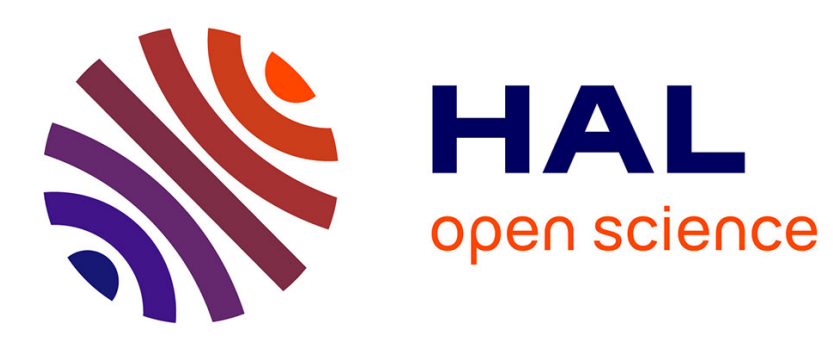

\title{
Optimal decay rates for the stabilization of a string network
}

\author{
Mohamed Jellouli, Michel Mehrenberger
}

\section{To cite this version:}

Mohamed Jellouli, Michel Mehrenberger. Optimal decay rates for the stabilization of a string network.

Comptes Rendus. Mathématique, 2014, 10.1016/j.crma.2014.03.023 . hal-00977345

\section{HAL Id: hal-00977345 \\ https://hal.science/hal-00977345}

Submitted on 11 Apr 2014

HAL is a multi-disciplinary open access archive for the deposit and dissemination of scientific research documents, whether they are published or not. The documents may come from teaching and research institutions in France or abroad, or from public or private research centers.
L'archive ouverte pluridisciplinaire HAL, est destinée au dépôt et à la diffusion de documents scientifiques de niveau recherche, publiés ou non, émanant des établissements d'enseignement et de recherche français ou étrangers, des laboratoires publics ou privés. 


\title{
Optimal decay rates for the stabilization of a string network
}

\author{
Mohamed Jellouli ${ }^{\mathrm{a}}$, Michel Mehrenberger ${ }^{\mathrm{b}}$ \\ a Département de Mathématiques, Faculté des Sciences de Monastir, 5019 Monastir, Tunisie \\ b Institut de Recherche Mathématique Avancée, Université de Strasbourg, 7 rue René Descartes F-67084 Strasbourg, \\ France \\ Received $* * * * *$; accepted after revision +++++ \\ Presented by
}

\begin{abstract}
We study the decay of the energy for a degenerate network of strings[1], and obtain optimal decay rates when the lengths are all equal. We also define a classical space semi-discretization and compare the results with the exact method introduced in [3,4]. To cite this article: A. Name1, A. Name2, C. R. Acad. Sci. Paris, Ser. I 340 (2005).

Résumé

Taux de décroissance optimaux pour la stabilisation d'un réseau de cordes On étudie la décroissance de l'énergie pour un réseau de cordes dégénéré [1], et on obtient des taux de décroissance optimaux lorsque les longueurs sont égales. On définit aussi un semi-discrétisation classique et comparons les résultats avec la méthode exacte introduite dans [3,4]. Pour citer cet article: A. Name1, A. Name2, C. R. Acad. Sci. Paris, Ser. I 340 (2005).
\end{abstract}

\section{Introduction}

We recall that the dissipation condition $\partial_{x} u(t, 0)=\alpha \partial_{t} u(t, 0)$ at the origin of a vibrating elastic string fixed at its end point, stabilizes this string. More precisely, if $E(t)$ denotes the energy of the solution of the wave equation $\partial_{t}^{2} u(t, x)-\partial_{x}^{2} u(t, x)=0$ on $(0, \ell)$ subject to the initial condition $u(0,)=$.$a and$ $\partial_{t} u(0,)=$.$b , then E(t) \leq C e^{-\gamma_{\alpha} t}$ where $\gamma_{\alpha}=\frac{1}{\ell} \log \left|\frac{1+\alpha}{1-\alpha}\right|$. Moreover, we remark that if $\alpha=1$, then $E(t)=0, \forall t \geq 2 \ell$. Thus, the value 1 is the best choice of $\alpha$ that makes the system is an equilibrium state. The situation is completely different in the case when we consider a network of strings.

The problem of stabilization of nondegenerate network of strings was studied by K. Ammari, M. Jellouli

Email addresses: mohamed.jellouli@fsm.rnu.tn (Mohamed Jellouli), mehrenbe@math.unistra.fr (Michel Mehrenberger).

Preprint submitted to the Académie des sciences

March 20, 2014 
and M. Khenissi in [1], [2] and [3]. When the strings are coupled at a common end in a star-shaped configuration, it is proven in [2] that the solutions are not exponential stable in the energy space, in the nondegenerate case. In the particular case of two strings, which is equivalent to the pointwise stabilisation of one string, such a problem has been studied in [5]; exponential stabilization is obtained if and only if the lengths satisfy $\frac{\ell_{1}}{\ell 1+\ell_{2}}=\frac{p}{q}$, with $p$ and $q$ odd numbers, and the best decay rate, when fixing the total length $\ell_{1}+\ell_{2}$ is obtained when the lengths are equal $\left(\ell_{1}=\ell_{2}=\ell\right)$, with best decay rate $\gamma=\frac{\ln (3)}{\ell}$. Such problem has been also considered in [7], with different boundary conditions; in the degenerate case, the energy limit was identified and it was proven that the solution decays exponentially to that limit.

We consider here the case of a degenerate network of vibrating elastic strings when the pointwise feedback acts in the root of the tree (tree shaped network). Note that in the nondegenerate case, it is proven in [1] that the solutions are not exponential stable in the energy space. We calculate the limit energy $E_{\infty}:=\lim _{t \rightarrow+\infty} E(t)$ and we show that the decrease from $E(t)$ to $E_{\infty}$ is exponential, giving the best decay rate, when the lengths are equal. Finally we give numerical results in Section 3, which confirm the theoretical results.

\section{2. $E_{\infty}$ and best decay rate}

Let $N \geq 3$. We consider the initial data $\left(\left(a_{j}\right)_{1 \leq j \leq N},\left(b_{j}\right)_{1 \leq j \leq N}\right) \in \mathcal{H}:=\prod_{j=1}^{N} H^{2}\left(0, \ell_{j}\right) \times \prod_{j=1}^{N} H^{1}\left(0, \ell_{j}\right)$, satisfying the compatibility conditions

$$
a_{1}^{\prime}(0)=\alpha b_{1}(0), a_{1}^{\prime}\left(\ell_{1}\right)=\sum_{j=2}^{N} a_{j}^{\prime}(0) \text { and } a_{j}\left(\ell_{j}\right)=0, a_{1}\left(\ell_{1}\right)=a_{j}(0), j=2, \ldots, N,
$$

and the system of partial differential equations

$$
(S): \begin{cases}\partial_{t}^{2} u_{j}(t, x)-\partial_{x}^{2} u_{j}(t, x)=0, & t>0, x \in\left(0, \ell_{j}\right), j=1, \ldots, N, \\ u_{j}(t, 0)=u_{1}\left(t, \ell_{1}\right) \text { and } u_{j}\left(t, \ell_{j}\right)=0, & t \geq 0, j=2, \ldots, N, \\ \partial_{x} u_{1}(t, 0)=\alpha \partial_{t} u_{1}(t, 0), & t \geq 0(\alpha>0), \\ \partial_{x} u_{1}\left(t, \ell_{1}\right)=\sum_{j=2}^{N} \partial_{x} u_{j}(t, 0), & t \geq 0, \\ u_{j}(0, x)=a_{j}(x), \partial_{t} u_{j}(0, x)=b_{j}(x), x \in\left[0, \ell_{j}\right], j=1, \ldots, N .\end{cases}
$$

We define the energy of the solution of $(S)$ by

$$
E(t)=\frac{1}{2} \sum_{j=1}^{N}\left\|\partial_{t} u_{j}(t)\right\|_{L^{2}\left(0, \ell_{j}\right)}^{2}+\frac{1}{2} \sum_{j=1}^{N}\left\|\partial_{x} u_{j}(t)\right\|_{L^{2}\left(0, \ell_{j}\right)}^{2}
$$

and we prove the following theorems in the case where $\ell_{j}=\ell, j=1, \ldots, N$ (we denote by \|\| the norm of $\left.L^{2}(0, \ell)\right)$ :

Theorem 2.1 If $\left(\left(a_{j}\right)_{1 \leq j \leq N},\left(b_{j}\right)_{1 \leq j \leq N}\right) \in \mathcal{H}$ verify (1), then the energy limit is given by

$$
E_{\infty}=\frac{1}{2(N-1)} \sum_{j=2}^{N} \sum_{k=j+1}^{N}\left(\left\|\left(a_{k}^{\prime}-a_{j}^{\prime}\right)\right\|^{2}+\left\|\left(b_{k}-b_{j}\right)\right\|^{2}\right) .
$$


Now, we denote by $\alpha_{0}=\frac{2 \sqrt{N-1}}{N}, \Delta=\frac{4\left(\alpha^{2} N^{2}-4 N+4\right)}{(\alpha+1)^{2}}$ and $\lambda=-\frac{\frac{2(N-2)}{\alpha+1}+\sqrt{\Delta}}{2 N} .1$

Theorem 2.2 There is a constant $C=C_{N}(\alpha)>0$ such that for any initial data $\left(\left(a_{j}\right)_{1 \leq j \leq N},\left(b_{j}\right)_{1 \leq j \leq N}\right) \in$ $\mathcal{H}$ satisfying (1) the following inequality holds

$$
\begin{aligned}
& 0 \leq E(t)-E_{\infty} \leq C\left(\left\|a_{1}^{\prime}\right\|^{2}+\left\|b_{1}\right\|^{2}+\left\|\sum_{j=2}^{N} a_{j}^{\prime}\right\|^{2}+\left\|\sum_{j=2}^{N} b_{j}\right\|^{2}\right) \frac{e^{-\gamma t}}{|\Delta|}, \text { if } \alpha \neq \alpha_{0}, \\
& 0 \leq E(t)-E_{\infty} \leq C\left(\left\|a_{1}^{\prime}\right\|^{2}+\left\|b_{1}\right\|^{2}+\left\|\sum_{j=2}^{N} a_{j}^{\prime}\right\|^{2}+\left\|\sum_{j=2}^{N} b_{j}\right\|^{2}\right) t^{2} e^{-\gamma_{0} t}, \text { if } \alpha=\alpha_{0},
\end{aligned}
$$

where $\gamma=\frac{1}{\ell} \log \frac{1}{|\lambda|}>0$ and $\gamma_{0}=\frac{1}{\ell} \log \frac{N+2 \sqrt{N-1}}{N-2}$. The best decay rate is achieved in the sense that for all $\alpha>0$, there exists an initial data such that (4) and (5) become an equivalence:

$$
E(t)-E_{\infty} \geq C e^{-\gamma t} \text {, or, for } \alpha=\alpha_{0}, E(t)-E_{\infty} \geq C t^{2} e^{-\gamma_{0} t} \text {, with } C>0,
$$

and $\forall \alpha \neq \alpha_{0}$, we have $\gamma<\gamma_{0}$.

Remark 1 The case $N=2$ (with $\ell_{1}, \ell_{2}$ not necessarily of same size) can be recasted into the case $N=1$ (with $\left.\ell=\ell_{1}+\ell_{2}\right)$.

The proofs of these theorems are based on the operator of type $\tau$ developed in [3] and [4] and will be detailed in [6]. In fact we recall that

$$
E(t)=E(0)-\alpha \int_{0}^{t}\left|\partial_{t} u_{1}(s, 0)\right|^{2} d s
$$

and that for all $t \geq 0, \partial_{t} u_{1}(t, 0)=\left(\mathcal{P} \circ \widetilde{\mathcal{P}}^{-1}\right) F(t)+\frac{1}{\alpha+1}\left(\mathcal{L}_{\beta, \ell, 1}^{+} a_{1}^{\prime}+\mathcal{L}_{\beta, \ell, 1}^{-} b_{1}\right), \beta=\frac{\alpha-1}{\alpha+1}$.

In a first step, we evaluate the caracteristic elements of $\widetilde{\mathcal{P}}^{-1}$ and we prove that $\widetilde{\mathcal{P}}^{-1} \equiv(n, \delta)$, where $n: n(j)=2(j-1) \ell, j \geq 1$ and in the case $\alpha \neq \alpha_{0}$

$$
\delta: \delta(j)=a(\beta) \lambda^{j-2}+b(\beta) \mu^{j-2}, j \geq 2
$$

with $\mu=-\frac{\frac{2(N-2)}{\alpha+1}-\sqrt{\Delta}}{2 N}, a(\beta)=\frac{1}{N} \frac{(1-\lambda)(\beta-\lambda)}{\lambda-\mu}$ and $b(\beta)=-\frac{1}{N} \frac{(1-\mu)(\beta-\mu)}{\lambda-\mu}$. In the case $\alpha=\alpha_{0}$,

$$
\delta(j)=\left[\delta(2)+\left(\frac{1}{\lambda_{0}} \delta(3)-\delta(2)\right)(j-2)\right] \lambda_{0}^{j-2}, \forall j \geq 2 .
$$

In the second stage we calculate $\int_{0}^{2 n \ell}\left|\partial_{t} u_{1}(s, 0)\right|^{2} d s$, what will allow to find $E_{\infty}$ :

$$
\begin{aligned}
E_{\infty} & =\frac{1}{2} \sum_{j=2}^{N}\left(\left\|a_{j}^{\prime}\right\|^{2}+\left\|b_{j}\right\|^{2}\right)-\frac{1}{4(N-1)}\left(\left\|\sum_{j=2}^{N}\left(a_{j}^{\prime}-b_{j}\right)\right\|^{2}+\left\|\sum_{j=2}^{N}\left(a_{j}^{\prime}+b_{j}\right)\right\|^{2}\right) \\
& =\frac{1}{2(N-1)} \sum_{j=2}^{N} \sum_{k=j+1}^{N}\left(\left\|\left(a_{k}^{\prime}-a_{j}^{\prime}\right)\right\|^{2}+\left\|\left(b_{k}-b_{j}\right)\right\|^{2}\right) .
\end{aligned}
$$

In the end, we estimate the difference $E(t=2 n \ell)-E_{\infty}$ and we show the inequality (4) and (5).

1. $\sqrt{\Delta}=i \sqrt{-\Delta}$ if $\Delta<0$. Note that, $\Delta<0$ (resp. $\Delta=0$ ) if and only if $\alpha \in] 0, \alpha_{0}\left[\right.$ (resp. $\alpha=\alpha_{0}$ ) 


\section{Semi-discretization and numerical results}

Let $N_{j}, j=1, \ldots, d$ be positive integers. We define $h=\left(h_{1}, \ldots, h_{d}\right)$, with $h_{j}=\frac{\ell_{j}}{N_{j}}, j=1, \ldots, d$. We suppose that $\alpha>0$ and consider the following classical finite difference space semi-discretization of (S):

$$
\left\{\begin{array}{l}
u_{j, k}^{\prime \prime}(t)-\frac{u_{j, k-1}(t)-2 u_{j, k}(t)+u_{j, k+1}(t)}{h_{j}^{2}}=0, k=1, \ldots, N_{j}-1, j=1, \ldots, d, \\
\frac{u_{1,1}(t)-u_{1,0}(t)}{h_{1}}=\alpha u_{1,0}^{\prime}(t), \\
\frac{u_{1, N_{1}}(t)-u_{1, N_{1}-1}(t)}{h_{1}}=\sum_{j=2}^{d} \frac{u_{j, 1}(t)-u_{j, 0}(t)}{h_{j}}, \\
u_{1, N_{1}}(t)=u_{j, 0}(t) \text { and } u_{j, N_{j}}(t)=0, j=2, \ldots, d, \\
u_{j, k}(0)=u_{j, k}^{0}, u_{j, k}^{\prime}(0)=u_{j, k}^{1}, j=1, \ldots, d, k=0, \ldots, N_{j}, t \in(0, \infty),
\end{array}\right.
$$

where the initial conditions satisfy for $s=0,1$, the compatibility conditions

$$
u_{1, N_{1}}^{s}=u_{k, 0}^{s}=\frac{\sum_{j=2}^{d} \frac{u_{j, 1}^{s}}{h_{j}}+\frac{u_{1, N_{1}-1}^{s}}{h_{1}}}{\sum_{j=1}^{d} \frac{1}{h_{j}}} \text { and } u_{k, N_{k}}^{s}=0, k=2, \ldots, d .
$$

The solution of (7) can be written in the form $V(t)=\exp (t A) \phi$, where $V=\left(U, U^{\prime}\right)^{t}$,

$$
U=\left(u_{1,0}, u_{1,1}, \ldots, u_{1, N_{1}-1}, u_{2,1}, \ldots, u_{2, N_{2}-1}, \ldots, u_{d, 1}, \ldots, u_{d, N_{d}-1}\right) \in \mathbb{R}^{M}
$$

and $\phi \in \mathbb{R}^{2 M}$. The semi-discrete energy is defined by

$$
E_{h, \phi}(t)=\frac{1}{2} \sum_{j=1}^{d} h_{j}\left(\sum_{k=1}^{N_{j}-1}\left|u_{j, k}^{\prime}(t)\right|^{2}+\sum_{k=0}^{N_{j}-1}\left|\frac{u_{j, k+1}(t)-u_{j, k}(t)}{h_{j}}\right|^{2}\right),
$$

and is decreasing as it satisfies $E_{h, \phi}^{\prime}(t)=-\alpha\left|u_{1,0}^{\prime}(t)\right|^{2}$. The following proposition identifies the limit of the discrete energy, which may not be zero, as in the continuous case.

Proposition 3.1 For $\phi \in \mathbb{R}^{2 M}$, we can write $\phi=\phi_{1}+\phi_{2}$ where $\phi_{1}$ belongs to the space $\Lambda_{1}$ of eigenvectors relative to the eigenvalues $\lambda$ of $A$, with $\Re(\lambda)=0$ and $\phi_{2}$ belongs to the space $\Lambda_{2}$ of generalized eigenvectors relative to the eigenvalues $\lambda$ of $A$, with $\Re(\lambda)<0$. We then have

$$
E_{h, \phi, \infty}:=\lim _{t \rightarrow \infty} E_{h, \phi}(t)=E_{h, \phi_{1}}(0),
$$

and this value does not depend on $\alpha>0$.

Sketch of the proof We first proof that for $\phi_{1} \in \Lambda_{1} \subset \mathbb{C}^{2 M}$, we have $\phi_{1, M+1}=0$, that no eigenvalue of $A$ can have positive real part and that generalized eigenvectors with eigenvalue of zero real part are necessarily eigenvectors, using the fact that the energy is decreasing and nonnegative. We then use the decomposition and estimates

$$
\begin{aligned}
& \left|E_{h, \phi}(t)-E_{h, \phi_{1}}(t)-E_{h, \phi_{2}}(t)\right|=\left|\sum_{j=1}^{d} \sum_{k=1}^{N_{j}-1} h_{j}\left(w_{j, k}^{1}\right)^{\prime}\left(w_{j, k}^{2}\right)^{\prime}+\sum_{j=1}^{d} \sum_{k=0}^{N_{j}-1} \frac{1}{h_{j}}\left(w_{j, k+1}^{1}-w_{j, k}^{1}\right)\left(w_{j, k+1}^{2}-w_{j, k}^{1}\right)\right| \\
& \leq \sum_{j=1}^{d} h_{j} \sqrt{\sum_{k=1}^{N_{j}-1}\left|\left(w_{j, k}^{1}\right)^{\prime}\right|^{2}} \sqrt{\sum_{k=1}^{N_{j}-1}\left|\left(w_{j, k}^{2}\right)^{\prime}\right|^{2}}+\frac{1}{h_{j}} \sqrt{\sum_{k=0}^{N_{j}-1}\left|w_{j, k+1}^{1}-w_{j, k}^{1}\right|^{2}} \sqrt{\sum_{k=0}^{N_{j}-1}\left|w_{j, k+1}^{2}-w_{j, k}^{2}\right|^{2}}
\end{aligned}
$$



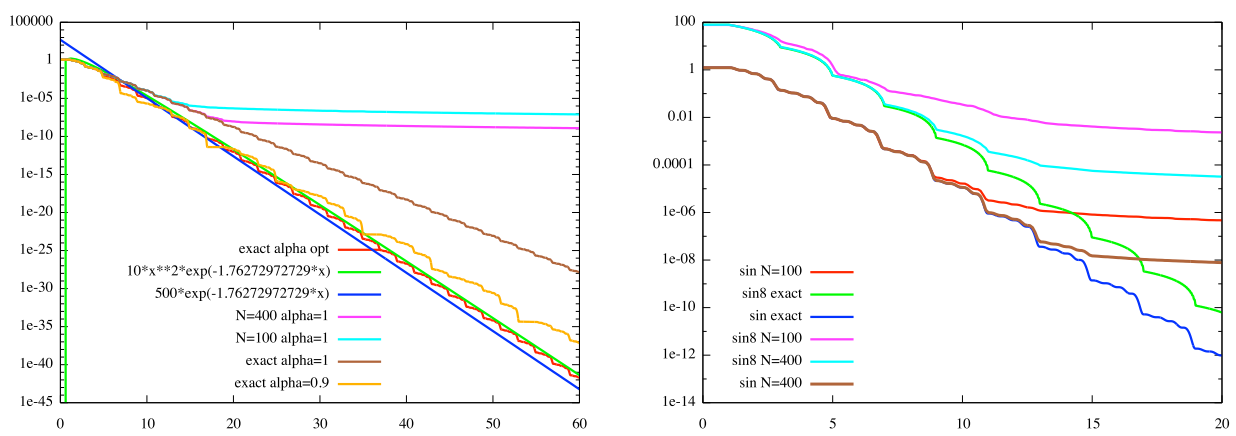

Figure 1. $E(t)-E_{\infty}$ vs time (exact and semi-discrete case) for $\alpha \in\left\{0.9,1, \alpha_{\mathrm{opt}}\right\}$ (left) and different initial conditions (right).

$$
\leq 2 d \sqrt{E_{h, \phi_{1}}(t)} \sqrt{E_{h, \phi_{2}}(t)},
$$

where $w_{j, k}^{1}$ (resp. $w_{j, k}^{2}$ ) is the solution corresponding to the initial condition $\phi_{1}$ (resp. $\phi_{2}$ ). We know that $E_{h, \phi_{1}}(t)=E_{h, \phi_{1}}(0)$, thus we get (9) since $E_{h, \phi_{2}}(t)$, being a finite sum and product of terms tending to zero, as $t \rightarrow+\infty$, also tends to zero. This limit does not depend on $\alpha$, since the only equation where $\alpha$ is present is

$$
\alpha \lambda \phi_{\lambda, M+1}=0=\frac{1}{h_{1}}\left(\phi_{\lambda, M+2}-\phi_{\lambda, M+1}\right), \text { for each } \lambda ; \Re(\lambda)=0 .
$$

Numerical results. Finally we give some exact and semi-discrete results for the energy. We take $d=3$, initial condition

$$
u_{j}^{0}(x)=0, j=1,3, u_{2}^{0}(x)=\sin ^{2}\left(\frac{\pi x}{\ell_{2}}\right) \text { and } u_{j}^{1}(x)=0, j=1,2,3 .
$$

$\ell_{j}=1$ and $N_{j}=N, j=1,2,3$. We see on Figure 1 (left) the time evolution of $E(t)-E_{\infty}$ for $\alpha=1$ (exact, $N=100,400$ ) and for $\alpha=\alpha_{\text {opt }}, 0.9$ (exact). On Figure 1 (right), we plot the time evolution of $E(t)-E_{\infty}$ (exact, $\left.N=100,400\right)$ taking as before $u_{2}^{0}(x)=\sin ^{2}(\pi x)$ (sin) but also $u_{2}^{0}(x)=\sin ^{2}(8 \pi x)$ $(\sin 8)$, in the case $\alpha=\alpha_{\text {opt }}$. The results are conform with Theorem 2.2: taking $\alpha=\alpha_{\text {opt }}$ leads to the best growth rate with asymptotic behaviour of the form $C t^{2} \exp (-\gamma t)$. We also note that the semi-discrete scheme is able to reproduce the behaviour of the exact scheme until the discretization error dominates, which is higher by taking a higher mode (Figure 1, right). The semi discrete energy seems to converge to a discretization dependent limit, which is coherent with Proposition 3.1.

\section{References}

[1] K. Ammari, M. Jellouli and M. Khenissi, Stabilization of Generic Tree of String. Journal of Dynamical and control System, Vol. 11, No. 2, April 2005, 177-193.

[2] K. Ammari and M. Jellouli, Stabilization of Star-Shaped Networks of Strings. Differential and Integral Equations, Vol. 17, No. 11-12, 2004, 1395-1410.

[3] K. Ammari and M. Jellouli, Remark on Stabilization of Tree-Shaped Networks of Strings. Application of Mathematics, Vol. 52, No. 4, 2007, 327-343.

[4] K. Ammari and M. Jellouli, Méthode numérique pour la décroissance de l'énergie d'un réseau de cordes. Bulletin of the Belgian Mathematical Society-Simon Stevin, 4 (2010), 717-735.

[5] K. Ammari, A. Henrot and M. Tucsnak, Asymptotic behaviour of the solutions and optimal location of the actuator for the pointwise stabilization of a string, Asymptotic Analysis., 28 (2001), 215-240. 
[6] M. Jellouli and M. Mehrenberger, Optimal decay rates for the stabilization of a string network, in preparation.

[7] S. Nicaise and J. Valein, A remark on the stabilization of the 1-d wave equation, C. R. Math. Acad. Sci. Paris, 348(1-2), 47-51, 2010. 